\title{
The utility of the sequential organ failure assessment score in predicting mortality in critically ill obstetric patients admitted to a high dependency unit in Uganda
}

\begin{abstract}
Background: Most maternal deaths result from severe maternal morbidity (SMM with subsequent organ/system dysfunction and organ failure. The objective was to assess the performance of Sequential Organ Failure Assessment (SOFA) score in cases of SMM admitted to the high dependency obstetric unit.

Methods: Organ/system dysfunction and failure were assessed according to the maximum score for the six components (respiratory, neurological, renal, hepatic, cardiovascular and coagulation system). The total maximum SOFA score was estimated using the worst result each component on a single day. The distribution of SOFA scores was assessed for normality by using the Shapiro Wilk Test, [ $\mathrm{p}=003$ ] The mean maximum SOFA scores for each organ/system were computed according to according to outcome (survival or death), using the Mann-Whitney test. The sensitivity, specificity, and the area under the curve (AUC) for each organ/system were evaluated. A Receiver Operator Characteristic (ROC) curve was fitted using maximum likelihood estimates for the total maximum SOFA score, in order to assess the sensitivity, specificity and discriminatory abilities of the maximum SOFA scores.

Results: Of the 425 patients with SMM, 345(81.2\%,95\%CI 71.8-82.4) survived while $80(18.8 \%, 95 \%$ CI: $12.9,22.6)$ did not survive. All the non-survivors and $64(18.5 \%)$ of the survivors presented with multiple organ/system dysfunction. Nonsurvivors were more likely to present with more severe and with multiple organ/ system dysfunction. The maximum total SOFA score had good discrimination in the respiratory, cardiovascular and neurological systems, but poor discrimination in the renal, hepatic and coagulatory systems. The total maximum SOFA score displayed an area under the curve (AUC) of $0.83(95 \% \mathrm{CI}: 0.56,1.00)$, with a cutoff value of at least 8.0 sensitivity of $86.7 \%$, and specificity of $90.0 \%$.
\end{abstract}

Conclusion: The total maximum SOFA score showed good predictive and discriminative abilities for maternal mortality in women with severe maternal morbidity.

Keywords: sofa score, severe maternal morbidity, maternal near miss
Volume 3 Issue 2 - 2017

\author{
Dan Kabonge Kaye,' Scovia Nalugo \\ Mbalinda $^{2}$ \\ 'Department of Obstetrics and Gynecology, Makerere \\ University, Uganda \\ ${ }^{2}$ Department of Nursing, Makerere University, Uganda
}

Correspondence: Dan K Kaye, Department of Obstetrics and Gynecology, School of Medicine, College of Health Sciences, Makerere University, P.O. Box 7072, Kampala, Uganda, Tel 2564|4-53436I, Fax 256-4|4-53345I, Email dankkaye@yahoo.com

Received: October 26, 2017 | Published: November 20, 2017
Abbreviations: AKI, acute kidney injury; ARDS, acute respiratory distress syndrome; AUC, area under the roc curve; HELLP syndrome, syndrome of hemolysis elevated liver enzymes and low platelets; HDU, high dependency obstetric unit; ICU, Intensive CARE UNIT; REC, Research and Ethics Committee; ROC, curve-receiver operator characteristic curve; SOFA, sequential organ failure assessment; SD, standard deviation; SMM, severe maternal morbidity; WHO, world health organization

\section{Introduction}

Obstetric complications are a major cause of maternal morbidity and mortality, where maternal death is a final point on a continuum between severe (though non-life-threatening) and fatal obstetric complications. ${ }^{1,2}$ On this continuum are patients with severe obstetric complications, severe maternal morbidity (SMM), who develop organ or system dysfunction. ${ }^{3,4}$ Some of these are referred to as maternal near miss in case they narrowly survive death from such severe complications. ${ }^{5-6}$ Both early recognition, detection and referral of potentially life threatening severe obstetric complications, and prompt access to safe, affordable basic and emergency obstetric care, are essential to reduce maternal morbidity and mortality. ${ }^{3-6}$

The challenge of predicting severe morbidity and mortality is that different obstetric patient populations tend to develop different patterns of organ system dysfunction. ${ }^{8-10}$ An ideal scoring system should allow both quantification of the severity of illness and estimation of the probability of mortality during hospitalization. ${ }^{8-11}$ The ideal scoring system would have the following characteristics: ${ }^{8-11}$ should be based on routinely collected data or variables, should be sensitive enough to identify all cases or possibly potential cases of organ/system dysfunction, and should be specific enough with a high level of discrimination. In addition, it should be objective, reliable, show abnormality in only one direction, and should be applicable to diverse obstetric population settings. Also, it should be reproducible, therapy independent and reflect acute rather than chronic organ 
dysfunction, with ability to predict quality of life and functional status for survivors beyond hospitalization. No scoring system is ideal.

The $\mathrm{SOFA}^{7,8}$ score was developed by a group from the European Society of Intensive Care Medicine to describe the degree of organ dysfunction associated with sepsis. It employs parameters from six organ systems-respiratory, cardiovascular, central nervous systems, renal, coagulation, and liver which are weighted (each 1-4) to give a final score [6-24 (maximum)]. The SOFA score has since then been validated to describe organ dysfunction in diverse patients with obstetric complications unrelated to sepsis ${ }^{9-11}$ and has been validated to quantify the severity and extent of organ dysfunction and to predict prognosis for severely ill patients. ${ }^{12-15}$ As the organ system functioning worsens, the score increases. SOFA scores can therefore assess individual or multiple organ dysfunction or failure.

Studies that have evaluated SMM in intensive care units (ICU) have reported that the degree of organ dysfunction, the number of failing organs, and the duration of the condition are directly related to higher maternal mortality. ${ }^{12-15}$ Prior studies have revealed high maternal morbidity and mortality related to severe obstetric complications in our setting. ${ }^{16,17}$ While withholding treatment in cases requiring ICU care increases maternal morbidity and mortality, unnecessary admissions lead to wastage of scanty resources and deny those who qualify the opportunity to receive necessary prompt care. ${ }^{18,19}$ However, there is little information from Uganda the patterns of organ dysfunction based on SOFA scores in severely ill obstetric patients. We hypothesized that scores that indicated organ/system dysfunction were correlated with mortality. If they were, the SOFA scores would be useful and reliable as proxy indicators of maternal near miss.

\section{Methods}

This study was conducted in Mulago hospital, the national referra hospital for Uganda. The detailed methods have been described elsewhere. ${ }^{16}$ Briefly the study involved women with severe maternal morbidity (SMM) admitted to the high dependency obstetric unit (HDU), between March 1, 2013 and January 28, 2014. Data was collected on age, parity, obstetric complication, management received, investigations done for organ/system dysfunction, referral for more specialized care (such dialysis for acute kidney injury (AKI), death and survival. Those patients who survived death (maternal near miss) after manifesting organ/system dysfunction were classified as maternal near miss.

With modification of the procedure described by Moreno et al. ${ }^{20}$ the maximum SOFA score was determined for each of the six organ systems- respiratory, coagulation, hepatic, cardiovascular, neurologic and renal to reflect the worst score during the entire duration of hospitalization in the ICU or HDU. Since arterial blood gas analysis was not routinely performed, most patients did not have records of the partial pressure of oxygen $\left(\mathrm{PaO}_{2}\right)$, and therefore the oxygen saturation level with a pulse oximeter or monitor was used as a proxy. Likewise, renal dysfunction is derived from serum creatinine levels, which were not routinely available on all days of hospitalization for all admitted patients. Diagnosis of renal dysfunction relied on the worst level of urine output, serum urea or serum electrolytes as surrogate markers of renal dysfunction. For all organ/systems, a maximum SOFA score of $\geq 1$ was used to define organ/system dysfunction, while a score of $\geq 3$ was used to diagnose organ/system failure. The aggregate total maximum SOFA score (range 0-24) was derived from the maximum SOFA score for each individual organ. Thus the maximum score was the worst score during the period of hospitalization.
For data analysis, the mean and standard deviation or medians with interquartile ranges were computed for numerical variables, while frequencies and percentages were computed for categorical variables. The distribution of SOFA scores was assessed for normality by using the Shapiro Wilk Test, $[p=003]$. The SOFA scores were not normally distributed. The median maximum SOFA scores for each organ/system evaluated were computed according to according to outcome (survival or death), using the Mann-Whitney nonparametric test for independent samples. For each system assessed, sensitivity, specificity, and the area under the curve (AUC) were evaluated, together with their respective $95 \%$ confidence intervals $(95 \% \mathrm{CI})$. Assuming a binormal distribution, a Receiver Operator Characteristic (ROC) curve with confidence bands was fitted using maximum likelihood estimates for the total maximum SOFA score, in order to assess the sensitivity, specificity and discriminatory abilities of the maximum SOFA scores to predict survival.

This research was part of a post-doctoral research project of the last author (DKK) entitled: "Evaluation and surveillance of the impact of maternal and neonatal near-miss morbidity on the health of mothers and infants in Jinja and Mulago hospitals". Ethical approval to conduct the study was obtained from the Ethics and research committees of Mulago hospital (REC 310-2012), the School of Medicine, Makerere University College of Health Sciences (REC 2012-172) and from Uganda National Council for Science and Technology. Permission to conduct the study was obtained from the department of Obstetrics and Gynaecology, Makerere University, and from Mulago National Referral Hospital and Jinja Hospital.

Participants gave written informed consent to be enrolled in the study and for their data to be included in the study. The languages used in the informed consent were English and the local languages (Luganda and Lusoga for Mulago and Jinja hospitals respectively). Participants included minors (aged 14-17years), as Uganda national guidelines for human subject research allow research on mature and emancipated minors in certain situations (such as in pregnancy), with prior approval of an institutional review board.

For those with very severe morbidity, consent was obtained retrospectively when they recovered, or consent was obtained from the next of kin to involve the patients' in the study and/or to include the patients' information in our data. Participants and their next of kin received assurances that participation was voluntary, and that participants were free to stop participation at any time without their decision affecting the care they were entitled to. All those with complications, and their newborns, were provided free medical care or, where necessary, were offered additional counseling or referred to get other support services not available at the two health facilities. Permission was obtained from the management of the two referral hospital (and from the study participants) to review the participants' records.

\section{Results}

The study included 425 women with severe obstetric complications, admitted to the high dependency unit of Mulago hospital, from March 1, 2013 to February 28, 2014). The maternal characteristics of the 425 women have been presented in Table 1 . The mean duration of stay in the HDU was 2.45 (2.12) days and it ranged from 1 to 13 days. Of the $425,345(81.2 \%, 95 \%$ CI $71.8-82.4)$ survived while $80(18.8 \%$, $95 \%$ CI: 12.9, 22.6) did not survive. All the non-survivors presented with multiple organ/system dysfunction, while $64(18.5 \%)$ of the survivors presented with multiple organ dysfunction. Table 2 shows a comparison of the maximum and total SOFA scores for women who 
survived with SMM and women who did not survive, showing that non-survivors were more likely to present with more severe and with multiple organ/system dysfunction.

Table I Socio-demographic characteristics and clinical diagnoses of the admitted severely ill patients

\begin{tabular}{|c|c|c|}
\hline Characteristics & $\begin{array}{l}\text { Number } \\
\text { (percentage) } \\
\text { Maternal Deaths } \\
\mathrm{n}=\mathbf{8 0} \mathrm{N} \%\end{array}$ & $\begin{array}{l}\text { Number } \\
\text { (percentage) } \\
\text { Maternal Near Miss } \\
\text { n=345 N \% }\end{array}$ \\
\hline \multicolumn{3}{|l|}{ Age Category } \\
\hline 18 years or less & $7(8.5)$ & $29(8.2)$ \\
\hline $19-24$ years & $30(36.9)$ & $125(36.1)$ \\
\hline$>=24$ years & $43(54.6)$ & $191(55.4)$ \\
\hline \multicolumn{3}{|l|}{ Gravidity } \\
\hline I & $18(22.3)$ & $71(20.5)$ \\
\hline 4-Feb & $38(47.7)$ & $195(56.4)$ \\
\hline 5 and more & $24(30.0)$ & $79(23.2)$ \\
\hline \multicolumn{3}{|l|}{ Education level } \\
\hline $\begin{array}{l}\text { None or primary } \\
\text { level }\end{array}$ & $45(56.3)$ & I 54 (44.7) \\
\hline Secondary level & $31(38.2)$ & I $64(47.5)$ \\
\hline $\begin{array}{l}\text { Post-secondary } \\
\text { (tertiary) }\end{array}$ & $4(5.5)$ & $27(6.6)$ \\
\hline \multicolumn{3}{|c|}{ Obstetric haemorrhage } \\
\hline Antepartum & $4(5.4)$ & $37(10.8)$ \\
\hline Postpartum & $22(26.9)$ & $85(24.7)$ \\
\hline Ruptured uterus & $17(20.8)$ & $58(16.5)$ \\
\hline None & $37(46.2)$ & $180(52.2)$ \\
\hline \multicolumn{3}{|l|}{ Abortion-related } \\
\hline Hemorrhage & $5(6.3)$ & $12(3.3)$ \\
\hline Postabortion sepsis & $0(0.0)$ & $3(0.9)$ \\
\hline Septic abortion & I (I.3) & $5(1.4)$ \\
\hline $\begin{array}{l}\text { Not abortion } \\
\text { related }\end{array}$ & $76(89.4)$ & $325(94.4)$ \\
\hline \multicolumn{3}{|c|}{ Hypertensive disorders } \\
\hline Severe Preeclampsia & $3(3.8)$ & $84(24.3)$ \\
\hline Eclampsia & $8(10.0)$ & $62(18.0)$ \\
\hline $\begin{array}{l}\text { Chronic } \\
\text { Hypertension }\end{array}$ & $0(0.0)$ & $4(1.2)$ \\
\hline HELLP Syndrome & $2(1.5)$ & $7(2.0)$ \\
\hline $\begin{array}{l}\text { Not hypertension } \\
\text { related }\end{array}$ & $67(84.0)$ & $195(56.4)$ \\
\hline \multicolumn{3}{|l|}{ Pueperal sepsis } \\
\hline Present & $9(10.8)$ & $42(\mathrm{II} .2)$ \\
\hline $\begin{array}{l}\text { Not related to } \\
\text { sepsis }\end{array}$ & $71(89.2)$ & $303(89.8)$ \\
\hline \multicolumn{3}{|l|}{ Obstructed labor } \\
\hline Present & $12(14.6)$ & $82(22.7)$ \\
\hline $\begin{array}{l}\text { Not related to } \\
\text { obstructed labor }\end{array}$ & $68(85.4)$ & $263(77.8)$ \\
\hline
\end{tabular}

Table $3 \&$ Table 4 show the ability of the maximum SOFA score, evaluated for each of the individual organ/system, to predict or discriminate between survivors and survivors. The maximum total SOFA score had good discrimination in the respiratory, cardiovascular and neurological systems, but poor discrimination in the renal, hepatic and coagulatory systems. Table 5 shows the ability of the total SOFA score to predict or discriminate survival in critically ill obstetric patients. The total maximum SOFA score displayed an area under the curve (AUC) of 0.83 (95\% CI: $0.56,1.00)$, with a cutoff value of at least 8.0 sensitivity of $86.7 \%$, and specificity of $90.0 \%$.

Table 2 Comparison of the maximum SOFA scores between survivors and non survivors admitted with severe maternal morbidity for different organ/ system dysfunction

\begin{tabular}{llll}
$\begin{array}{l}\text { System } \\
\text { involved }\end{array}$ & $\begin{array}{l}\text { Maternal } \\
\text { death } \\
\text { (Mean } \pm \text { SD) }\end{array}$ & $\begin{array}{l}\text { Maternal near } \\
\text { miss } \\
\text { (Mean } \pm \text { SD) }\end{array}$ & $\begin{array}{l}\text { p-value } \\
\text { (Wilcoxon } \\
\text { rank test) }\end{array}$ \\
\hline Respiratory & $5.0(3.3)$ & $1.5(0.8)$ & $<0.001$ \\
Coagulation & $1.6(1.3)$ & $1.5(1.0)$ & 0.448 \\
Hepatic & $2.9(1.2)$ & $2.0(1.4)$ & $<0.001$ \\
Cardiovascular & $3.2(0.7)$ & $1.9(1.2)$ & $<0.001$ \\
Neurologic & $2.4(0.7)$ & $1.8(2.0)$ & 0.009 \\
Renal & I.4 (0.7) & $0.8(1.0)$ & $<0.001$ \\
$\begin{array}{l}\text { Total maximum } \\
\text { SOFA Score }\end{array}$ & SI5.7(5.8) & $4.8(1.8)$ & $<0.001$ \\
\hline
\end{tabular}

Table 3 Sensitivity, specificity and area under the curve (AUC) for the receiver operator characteristic (ROC) curve of individual SOFA scores in prediction of survival

Score Sensitivity Specificity \begin{tabular}{l}
$\begin{array}{l}\text { Area under the } \\
\text { ROC curve }\end{array}$ \\
\hline
\end{tabular}

\section{Respiratory system}

$\begin{array}{llll}\geq 1 & 100 & 56.8 & 0.90(95 \% \mathrm{Cl}: 0.82, \\ \geq 2 & 100 & 69.9 & 1.00) \\ \geq 3 & 80 & 78.3 & \\ \geq 4 & 80 & 94.2 & \end{array}$

\section{Neurological system}

$\begin{array}{llll}\geq 1 & 60 & 71.88 & 0.70(95 \% \mathrm{Cl}: 0.42, \\ \geq 2 & 40 & 89.06 & 0.99) \\ \geq 3 & 40 & 98.44 & \\ \geq 4 & 40 & 100 \mathrm{~s} & \end{array}$

\section{Cardiovascular $\quad 0.65(95 \% \mathrm{Cl} 0.40$}

system 0.90$)$

$\begin{array}{lll}\geq 2 & 40 & 89.06 \\ \geq 3 & 40 & 96.88 \\ \geq 4 & 20 & 100\end{array}$


Table 4 Sensitivity, specificity and area under the curve (AUC) for the receiver operator characteristic (ROC) curve of individual SOFA scores that did not predict survival

\begin{tabular}{llll}
\hline Score & Sensitivity & Specificity & $\begin{array}{l}\text { Area under the ROC } \\
\text { curve }\end{array}$ \\
\hline $\begin{array}{l}\text { Hepatic system } \\
\geq 1\end{array} \quad 60$ & 55.6 & \\
$\geq 2$ & 20 & 64.3 & $0.56(95 \% \mathrm{Cl}: 0.28,0.80)$ \\
$\geq 3$ & 20 & 86.5 & \\
$\geq 4$ & 0 & 98.8 & \\
Renal system & & \\
$\geq 1$ & 40 & 70.31 & $0.58(95 \% \mathrm{Cl} 0.30,0.72)$ \\
$\geq 2$ & 40 & 87.5 & \\
$\geq 3$ & 0 & 98.44 & \\
Coagulation system & & \\
$\geq 1$ & 80 & 29.69 & \\
$\geq 2$ & 60 & 43.75 & \\
$\geq 3$ & 0 & 73.44 & \\
\hline
\end{tabular}

Table 5 Sensitivity and Specificity of total SOFA scores in predicting maternal morbidity in women with SMM (Area under the curve 0.83 (95\% Cl: 0.56 , $1.00)$

\begin{tabular}{lll}
\hline $\begin{array}{l}\text { Total maximum SOFA } \\
\text { Score }\end{array}$ & Sensitivity & Specificity \\
\hline$\geq 1$ & 100 & 7.81 \\
$\geq 2$ & 100 & 12.5 \\
$\geq 3$ & 80 & 23.44 \\
$\geq 4$ & 80 & 32.81 \\
$\geq 5$ & 80 & 48.44 \\
$\geq 6$ & 80 & 60.94 \\
$\geq 7$ & 60 & 78.13 \\
$\geq 8$ & 60 & 84.38 \\
$\geq 9$ & 60 & 90.63 \\
$\geq 10$ & 60 & 98.44 \\
$\geq 12$ & 60 & 98.44 \\
$\geq 14$ & 20 & 100 \\
\hline
\end{tabular}

\section{Discussion}

The study shows that the total maximum SOFA score had good predictive and discriminative abilities for survival or death in critically ill obstetric patients. Assessment of organ/system dysfunction is essential in the management of severe maternal morbidity (SMM). ${ }^{6-8,21-24}$ SMM refers to women with severe obstetric complications, some of whom die and others narrowly survive death (maternal near miss). ${ }^{25}$ Scores based on criteria of organ/system dysfunction or failure, such as SOFA scores, have greater discriminatory power in cases of SMM. ${ }^{6,7,9,14,21}$ Consequently, women who survive SMM after presenting with high SOFA scores may be classified as maternal near miss, as the high SOFA scores represent severe organ/system dysfunction or failure ${ }^{1,11-15}$ during their hospitalization.

In this study, the modified maximum SOFA score enabled analysis of the pathophysiologic process of SMM, as the aggregated score reflects most severe degree of organ/system dysfunction. Apparently, the maximum SOFA score performs better than a score for each individual organ/system dysfunction. This could be explained by the interdependence of organ/system function and the fact that several organ/systems are simultaneously or consequently involved during critical obstetric illness. ${ }^{16}$ Such multiple organ/system involvement may be subtle or unrecognized.

The commonest clinical diagnoses Most patients in our study participants were severe preeclampsia and eclampsia, obstetric hemorrhage, ruptured uterus and severe obstructed labor. These diagnoses may differ in different countries. This constitutes a challenge in predicting survival and mortality as different obstetric patient populations may develop different patterns of organ/system dysfunction. $^{8-10}$ This challenge necessitates identification and validation of population-specific scores for organ/system dysfunction in patients with obstetric morbidity. The SOFA score offers several advantages:26-30 it uses variables that can be easily measured without the need of very complex resources, proxy measures could be used in cases where complex or expensive variables cannot be measured, measurements can easily be standardized, and scores have high predictive and discriminatory ability in SMM. As in other studies, ${ }^{12-14,21-24,26}$ the maximum aggregated SOFA scores were prognostic, though in retrospect. In our study, the $95 \%$ confidence limits of the point estimates for the area under ROC curves of nearly all the systems showed that upper limits were $>0.75$ for all organs, indicates that severe obstetric morbidity is associated with multiple organ/system dysfunction. Indeed values of $\geq 10$ or $\geq 12$ significantly increase the probability of maternal deaths. ${ }^{12-14,21-24}$

This study evaluated the performance of total maximum SOFA score for cases of SMM and shows that the score has good performance. Assessment of the SOFA scores has potential utility in cases of SMM. ${ }^{5,10,11,13}$ The total SOFA score may be used to develop a cut off for referring obstetric patients with severe morbidity to referral units or to the HDU or ICU. Any score $>0$ as a cut-off point may be used for referral from a primary care centre, while an individual organ score of $\geq 1$ should referral to HDU or ICU. Individual organ SOFA scores $\geq 1$ or total SOFA score is $\geq 3$ indicates possibility of multiple organ dysfunction. Very high scores are suggestive of very severe organ/system dysfunction.

Organ-specific-based scoring systems such as the SOFA are superior to diagnosis-based systems for several additional reasons..$^{8-10}$ Many patients have simultaneous multiple organ/system dysfunction or failure during the course of severe obstetric illness, as demonstrated in our participants. Secondly, as shown by the reason for admission, obstetric patients may have multiple clinical diagnoses, either at admission or during their hospitalization. For instance, some patients admitted with postpartum hemorrhage later developed hypotension, shock, acute kidney injury or disseminated intravascular coagulopathy. Also, all patients with HELLP syndrome developed thrombocytopenia and acute kidney injury (AKI). Furthermore, severity scoring systems such as the SOFA allow generation of a score that reflects the severity of the condition that necessitates admission for critical care, thereby enabling comparison of patients with differing diagnoses as long as 
the scores are standardized..$^{8-10}$ If serial SOFA scores are generated over time, they could be used to monitor the patients' prognosis and response to therapy. In addition, the SOFA could be used as a tool to audit the quality of care. ${ }^{8-10}$

Nevertheless, SOFA score as evaluated in this study have some limitations. Their main purpose is to describe the sequence of complications (in SMM) and not to predict mortality, much as there is a relationship between organ/system dysfunction, failure and death. ${ }^{31}$ Some of the patients with multiple organ dysfunction in our study survived, yet others with lesser organ involvement did not. In addition, some laboratory-based variables were not routinely available on a daily basis. In spite of this, our findings are in agreement with previous research that SOFA scores have good discrimination between survivors and non-survivors. ${ }^{32}$ In addition, as in prior research, ${ }^{33-36}$ multiple organ dysfunction evidenced by high SOFA scores are associated with higher mortality. The use of proxy variables for SOFA scores has been validated in prior research. For instance, pulse oximetry as a measure of oxyhemoglobin saturation has been validated as a reliable alternative to measurement of $\mathrm{PaO} 2$ in calculation of the $\mathrm{SPO}_{2} / \mathrm{FiO}_{2} \cdot{ }^{36}$ Also, respiratory SOFA scores obtained using the SF ratio and the score calculated with the PF are well correlated. ${ }^{37}$ Another limitation of our study is that only the worst scores were used, yet in the course of critical illness, the severity of organ/system dysfunction may change over time.$^{38}$ Likewise, dynamic assessment of the SOFA score is superior at predicting 28-day survival/mortality. ${ }^{39-41}$ Furthermore, modifications of the SOFA score ${ }^{42-46}$ have been shown to have good discrimination of development of more severe morbidity.

\section{Conclusion}

Our results show that the total maximum SOFA score showed good and acceptable performance in patients with severe maternal morbidity admitted to the high dependency unit. The modified SOFA score was able to evaluate, though retrospectively, the severity and prognosis of morbidity by discriminating between survivors and non-survivors. The discriminatory power of the modified SOFA score seems this unaffected by the physiological changes that occur in pregnancy or the diversity of pregnancy complications that are associated with severe morbidity. Maximum values of the modified SOFA score may be used to conceptually define a maternal near miss. The results of the modifications of the SOFA score show the feasibility of using the SOFA scores in low resources settings.

\section{Acknowledgements}

This study was part of a post-doctoral research project funded by the Swedish International Development Agency (SIDA) through the Makerere University-Karolinska Institutet postdoctoral-research grants. The conclusions are those of the authors and do not necessarily represent the views of the funders or of Makerere University. We are grateful to all women who participated in this study

\section{Funding}

Funding for this research was provided by SIDA through Makerere University -Karolinska Institutet Research Collaboration postdoctoral research grant to Makerere University.

\section{Competing interests}

The authors declare that they have no competing interests.

\section{Availability of data and materials}

Data from is available on request from Makerere University research repository, through the authors.

\section{Consent for publication}

No individual identifier information is included in the manuscript. So consent for publication is not applicable.

\section{Ethics approval and consent to participate}

Ethical approval to conduct the study was obtained from the ethics and research committees of Mulago hospital (REC 310-2012), the School of Medicine, Makerere University College of Health Sciences (REC 2012-172) and from Uganda National Council for Science and Technology. Permission to conduct the study was obtained from the Department of Obstetrics and Gynaecology, Makerere University, and from Mulago National Referral Hospital and Jinja Hospital. Participants gave written informed consent to be enrolled in the study and for their data to be included in the study.

\section{Author's contributions}

DKK conceptualized the study as part of his post-doctoral research project. DKK collected the data, led the analysis, and wrote the text of the paper. SNM gave advice on the data analysis, presentation of the results, reviewed and edited the text and approved the final manuscript.

\section{References}

1. Mantel GD, Buchmann E, Rees H, et al. Severe acute maternal morbidity: a pilot study of a definition for a near-miss. British Journal of Obstetrics and Gynaecology. 1998;105(9):985-990.

2. Geller SE, Rosenberg D, Cox SM, et al. The continuum of maternal morbidity and mortality: factors associated with severity. Am J Obstet Gynecol. 2004;191(3):939-944.

3. Karnad DR, Lapsia V, Krishnan A, et al. Prognostic factors in obstetric patients admitted to an Indian intensive care unit. Critical Care Medicine. 2004;32(6):1294-1299.

4. Afessa B, Green B, Delke I, et al. Systemic inflammatory response syndrome, organ failure, and outcome in critically ill obstetric patients treated in an ICU. Chest. 2001;120(4):1271-1277.

5. Munnur U, Karnad DR, Bandi VDP, et al. Critically ill obstetric patients in an American and an Indian public hospital: comparison of case-mix, organ dysfunction, intensive care requirements, and outcomes. Intensive Care Medicine. 2005;31(8):1087-1094.

6. Karnad DR, Guntupalli KK. Critical illness and pregnancy: review of a global problem. Critical Care Clinics. 2004;20(4):555-576.

7. American College of Chest Physicians/Society of Critical Care Medicine. Consensus Conference: definitions for sepsis and organ failure and guidelines for the use of innovative therapies in sepsis. Critical Care Medicine. 1992;20(6):864-874.

8. Vincent JL, Moreno R, Takala J, et al. The SOFA (Sepsis-related Organ Failure Assessment) score to describe organ dysfunction/failure. Intensive Care Med. 1996;22(7):707-710.

9. Vincent JL, Moreno R. Clinical review: Scoring systems in the critically ill. Critical Care. 2010;14(2):207.

10. Bouch CD, Thompson JP. Severity scoring systems in the critically ill. Continuing Education in Anaesthesia, Critical Care \& Pain 2008;8(5):181-185. 
11. Kallur SD, Bada VP, Reddy P, et al. Organ Dysfunction and Organ Failure as Predictors of Outcomes of Severe Maternal Morbidity in an Obstetric Intensive Care Unit. J Clin Diagn Res. 2014;8(4):OC06-OC08.

12. Jain S, Guleria K, Suneja A, et al. Use of the Sequential Organ Failure Assessment score for evaluating outcome among obstetric patients admitted to the intensive care unit. Int $J$ Gynaecol Obstet. 2016;132(3):332-336.

13. Oliveira-Neto A, Parpinelli MA, Cecatti JG, et al. Sequential Organ Failure Assessment Score for Evaluating Organ Failure nd outcome of severe maternal morbidity in obstetric intensive care. Scientific World Journal. 2012;2012:172145.

14. Vasquez DN, Estenssoro E, Canales HS, et al. Clinical characteristics and outcomes of obstetric patients requiring ICU admission. Chest. 2007;131(3):718-724.

15. Pérez Assef A, Acevedo Rodríguez O, Del Consuelo Tamayo Gómez F, et al. Characterization of obstetric patients with multiple organ failure in the intensive care unit of a Havana Teaching Hospital, 1998 to 2006. MEDICC Review. 2010;12(2):27-32.

16. Nakimuli A, Nakubulwa S, Kakaire O, et al. Maternal near misses from two referral hospitals in Uganda: a prospective cohort study on incidence, determinants and prognostic factors. BMC Pregnancy Childbirth. 2016;16:24.

17. Okong P, Byamugisha J, Mirembe F, et al. Audit of severe maternal morbidity in Uganda--implications for quality of obstetric care. Actc Obstet Gynecol Scand. 2006;85(7):797-804

18. Mahutte NG, Murphy-Kaulbeck L, Le Q, et al. Obstetric admissions to the intensive care unit. Obstet Gynecol. 1999;94(2):263-266.

19. Selo-Ojeme DO, Omosaiye M, Battacharjee P, et al. Risk factors for obstetric admissions to the intensive care unit in a tertiary hospital: case-control study. Arch Gynecol Obstet. 2005;272(3):207-210.

20. Moreno R, Vincent JL, Matos R, et al. The use of maximum SOFA score to quantify organ dysfunction/failure in intensive care. Results of a prospective, multicentre study. Intensive Care Med. 1999;25(7):686-696.

21. Cecatti JG, Souza JP, Oliveira Neto AF. Pre-validation of the WHO organ dysfunction based criteria for identification of maternal near miss. Reproductive Health. 2011;8:22.

22. Hazelgrove JF, Price C, Pappachan VJ, et al. Multicenter study of obstetric admissions to 14 intensive care units in southern England. Critical Care Medicine. 2001;29(4):770-775.

23. Gilbert TT, Smulian JC, Martin AA, et al. Obstetric admissions to the intensive care unit: outcomes and severity of illness. Obstetrics and $G y$ necology. 2003;102(5):897-903.

24. Pérez Assef A, Acevedo Rodríguez O, Del Consuelo Tamayo Gómez F, et al. Characterization of obstetric patients with multiple organ failure in the intensive care unit of a Havana Teaching Hospital, 1998 to 2006. MEDICC Review. 2010;2(2):27-32.

25. Say L, Souza JP, Pattinson RC. Maternal near miss-towards a standard tool for monitoring quality of maternal health care. Best Practice \& Research: Clinical Obstetrics \& Gynaecology. 2009;23(3):287-296.

26. Şimsek T, Eyigor C, Uyar M, et al. Retrospective review of critically ill obstetrical patients: a decade's experience. Turk J Med Sci. 2011;41(6):1059-1064.

27. Soubra SH, Guntupalli KK. Critical illness in pregnancy: an overview. Critical Care Medicine. 2005;33(supplement 10):S248-S255.

28. Gopalan PD, Muckart DJJ. The critically ill obstetric patient: what's the score? International Journal of Obstetric Anesthesia. 2004;13(3):144-145.
29. Lopes Ferreira F, Peres Bota D, Bross A, et al. Serial evaluation of the SOFA score to predict outcome in critically ill patients. Journal of the American Medical Association. 2001;286(14):1754-1758.

30. Arts DGT, De Keizer NF, Vroom MB, et al. Reliability and accuracy of Sequential Organ Failure Assessment (SOFA) scoring. Critical Care Medicine. 2005;33(9):1988-1993.

31. Zeeman GG. Obstetric critical care: a blueprint for improved outcomes. Critical Care Medicine. 2006;34(supplement 9):S208-S214.

32. Lawton BA, Wilson LF, Dinsdale RA, et al. Audit of severe acute maternal morbidity describing reasons for transfer and potential preventability of admissions to ICU. Aust NZJ Obstet Gynaecol. 2010;(4):346-351.

33. Vincent JL, De Mendonca A, Cantraine F, et al. Use of the SOFA score to assess the incidence of organ dysfunction/failure in intensive care units: results of a multicenter, prospective study. Working group on "sepsis-related problems" of the European Society of Intensive Care Medicine. Crit Care Med. 1998;26:1793-1800.

34. Minne L, Abu-Hanna A, de Jonge E. Evaluation of SOFA-based models for predicting mortality in the ICU: A systematic review. Crit Care. 2008;12(6):R161

35. Regel G, Grotz M, Weltner T, et al. Pattern of organ failure following severe trauma. World J Surg. 1996;20(4):422-429.

36. Antonelli M, Moreno R, Vincent JL, et al. Application of SOFA score to trauma patients. Sequential Organ Failure Assessment. Intensive Care Med. 1999;25(4):389-394.

37. Moreno R, Vincent JL, Matos R, et al. The use of maximum SOFA score to quantify organ dysfunction/failure in intensive care. Results of a prospective, multicentre study. Working Group on Sepsis related Problems of the ESICM. Intensive Care Med. 1999;25(7):686-696.

38. MacIntyre N. Pulse oximetry in critical care scoring systems. Crit Care Med. 2009;37(4):1505-1506.

39. Pandharipande PP, Shintani AK, Hagerman HE, et al. Derivation and validation of Spo2/Fio2 ratio to impute for Pao2/Fio2 ratio in the respiratory component of the Sequential Organ Failure Assessment score. Crit Care Med. 2009;37(4):1317-1321.

40. Ferreira FL, Bota DP, Bross A, et al. Serial evaluation of the SOFA score to predict outcome in critically ill patients. JAMA. 2001;286(14):1754-1758.

41. Levy MM, Macias WL, Vincent JL, et al. Early changes in organ function predict eventual survival in severe sepsis. Crit Care Med. 2005;33(10):2194-2201.

42. Russell JA, Singer J, Bernard GR, et al. Changing pattern of organ dysfunction in early human sepsis is related to mortality. Crit Care Med. 2000;28(10):3405-3411.

43. Junger A, Engel J, Benson M, et al. Discriminative power on mortality of a modified Sequential Organ Failure Assessment score for complete automatic computation in an operative intensive care unit. Crit Care Med. 2002;30(2):338-342.

44. Grissom CK, Brown SM, Kuttler KG, et al. A modified sequential organ failure assessment score for critical care triage. Disaster Med Public Health Prep. 2010;4(4):277-284.

45. Baykara N, Gokduman K, Hosten T, et al. Comparison of sequentia organ failure assessment (SOFA) scoring between nurses and residents. J Anesth. 2011;25(6):839-844.

46. Jones AE, Trzeciak S, Kline JA. The Sequential Organ Failure Assessment score for predicting outcome in patients with severe sepsis and evidence of hypoperfusion at the time of emergency department presentation. Crit Care Med. 2009;37(5):1649-1654. 